Discrete Comput Geom 34:251-268 (2005)

DOI: $10.1007 / \mathrm{s} 00454-005-1170-6$

\title{
Criteria for Balance in Abelian Gain Graphs, with Applications to Piecewise-Linear Geometry*
}

\author{
Konstantin Rybnikov ${ }^{1}$ and Thomas Zaslavsky ${ }^{2}$ \\ ${ }^{1}$ Department of Mathematical Sciences, University of Massachusetts at Lowell, \\ Lowell, MA 01854, USA \\ krybniko@cs.uml.edu \\ ${ }^{2}$ Department of Mathematical Sciences, Binghamton University, \\ Binghamton, NY 13902-6000, USA \\ zaslav@math.binghamton.edu
}

\begin{abstract}
Consider a gain graph with abelian gain group having no odd torsion. If there is a basis of the graph's binary cycle space, each of whose members can be lifted to a closed walk whose gain is the identity, then the gain graph is balanced, provided that the graph is finite or the group has no non-trivial infinitely 2-divisible elements. We apply this theorem to deduce a result on the projective geometry of piecewise-linear realizations of cell-decompositions of manifolds.
\end{abstract}

\section{Introduction}

A gain graph $(\Gamma, g, G)$ consists of a graph $\Gamma=(V, E)$, a group $G$, and a homomorphism $g$ from the free group $F_{E}$ on the edges of $\Gamma$ to $G$. We call $g$ the gain map and $G$ the gain group. $^{1}$ (One thinks of the edges of $G$ as oriented in an arbitrary but fixed way.) Gain graphs have appeared in pure mathematics, physics, operations research, psychology, and discrete geometry, among many other areas (see Zaslavsky's annotated bibliography [26] for a survey).

\footnotetext{
* Part of the first author's research was conducted at Cornell University, Ithaca, NY 14853-4201, USA, and he was partially supported by the Mathematical Sciences Research Institute in Berkeley. The second author's research was partially supported by National Science Foundation Grant DMS-0070729.

${ }^{1}$ See, e.g., Section 5 of [25] for the basic theory of gain graphs. Gain graphs have been called voltage graphs in graph embedding theory-e.g., in [9]; we eschew the term "voltage" because gains do not have to obey Kirchhoff's voltage law.
} 
The simplest gain graphs are those in which every simple closed walk on $\Gamma$ lies in the kernel of $g$; such gain graphs are called balanced. If $\Gamma$ is connected, $(\Gamma, g, G)$ is balanced if and only if $\pi(\Gamma) \subseteq$ Ker $g$. Since for general gain graphs one can treat each component separately, we assume throughout that $G$ is connected. A basic problem is how to tell whether or not a gain graph is balanced. We investigate this problem when $G$ is abelian; then we call $(\Gamma, g, G)$ an abelian gain graph. We propose here a criterion for balance in abelian gain graphs that is broader than previous ones, but which has the drawback that it is not valid for all groups. Our topics are, first, the problem of describing the abelian gain groups for which it is valid and, second, an application of the criterion to a problem of polyhedral geometry.

To state the criterion we must first define a "circle" and a "cycle" in a graph. A circle is the edge set of a non-trivial simple closed walk (a walk in which no vertex or edge is repeated, except that the initial and final vertices are the same). There are two kinds

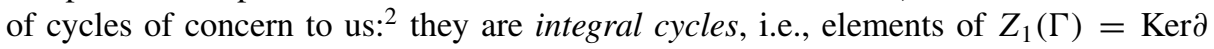
where $\partial$ is the standard one-dimensional boundary operator of cellular homology of $\Gamma$, and binary cycles, i.e., elements of $Z_{1}\left(\Gamma ; \mathbb{Z}_{2}\right)$. There are natural epimorphisms

$$
\pi(\Gamma) \stackrel{a}{\longrightarrow} Z_{1}(\Gamma) \stackrel{[]}{\longrightarrow} Z_{1}\left(\Gamma ; \mathbb{Z}_{2}\right)
$$

that consist, respectively, of abelianization and reduction of coefficients modulo 2 . Suppose that $b$ is a binary cycle: a cyclic orientation of $b$ is any closed walk $\widetilde{b}$ for which $\left[\tilde{b}^{a}\right]=b$. If $B$ is a set of binary cycles, a cyclic orientation of $B$ is any set $\widetilde{B}=\{\widetilde{b}: b \in B\}$ of cyclic orientations of the members of $B$. This definition is very broad: a binary cycle has many cyclic orientations; also, edges may appear in $\widetilde{b}$ that are not in the support of $b$, provided that each such edge appears an even number of times in $\widetilde{b}$. Thus, a binary cycle with disconnected support can have a cyclic orientation, which is always connected. In general, there is no cyclic orientation of a cycle that can reasonably be regarded as canonical, except in the case of a binary cycle whose support is a circle.

Now, our test for balance:

Definition. Let $(\Gamma, g, G)$ be a gain graph and let $B$ be a basis of $Z_{1}\left(\Gamma ; \mathbb{Z}_{2}\right)$. We say that $B$ passes the Binary Cycle Test if it has a cyclic orientation $\widetilde{B}$ such that all elements of $\widetilde{B}$ have gain 1 . We say the Binary Cycle Test is valid for $(\Gamma, g, G)$ if the existence of a basis $B$ that passes the Binary Cycle Test implies that $(\Gamma, g, G)$ is balanced. We say the Binary Cycle Test is valid for a family of graphs $\mathcal{G}$ and a family of groups $\mathcal{H}$ if it is valid for every gain graph $(\Gamma, g, G)$ with $\Gamma \in \mathcal{G}$ and $G \in \mathcal{H}$.

Our main results are that the Binary Cycle Test is valid for any abelian gain group that has no odd torsion if the graph is finite (Theorem 3.3) or if the gain group has no non-trivial infinitely 2-divisible elements (Theorem 3.5). We also show an infinite graph that fails the Binary Cycle Test for all gain groups that have an infinitely 2-divisible element other than the identity. (In [19] we prove that for any gain group with non-trivial elements of odd order there is a graph for which the Binary Cycle Test does not work.

\footnotetext{
2 The graph-theory literature contains at least five incompatible definitions of the term "cycle".
} 
Focussing on properties of the gain graph that are due to the gain group rather than the graph is how the present paper differs from that one.)

In most applications to physics, psychology, or operations research, the gain graphs are finite and have $\mathbb{Z}_{2}$ or $\mathbb{R}^{*}$ as the gain group. Groups $\mathbb{R}^{*}$ or $\mathbb{R}^{d}$ tend to appear in discretegeometry applications such as Voronoi's generatrix construction and the MaxwellCremona correspondence. The Binary Cycle Test applies to such situations, since all these groups are abelian and do not have odd torsion. However, if the gain graph is infinite, our results do not apply when $G=\mathbb{R}^{*}$ or $\mathbb{R}^{d}$, since these groups are 2-divisible. We know that for certain particular binary cycle bases their passing the Binary Cycle Test does imply balance even then, but they have to be carefully selected, as Example 4.1 shows.

The reason we need the Binary Cycle Test rather than a simpler test for balance of $(\Gamma, g, G)$, such as the existence of a set of balanced circles that form a fundamental system of circles (i.e., the set of circles obtained from a fixed spanning tree by adjoining each non-tree edge), is that, in applications, often a basis of the binary cycle space is given to us or is in some way naturally associated with the problem, so we are not free to choose the basis, or the geometry may yield information about the gains only of certain cycles that are not a fundamental system or otherwise tractable.

In Euclidean space, the sphere, or hyperbolic space, gain graphs are a natural setting for problems of existence and classification of tilings with prescribed restrictions on the group or the tiles, and of $(d+1)$-dimensional piecewise-linear realization of $d$-manifolds with given projection. Among important examples are Coxeter's theorem reducing the classification of reflection groups to that of Coxeter simplices [3], Alexandrov's spacefilling theorem [1], Voronoi's [23] construction of a generatrix (generatice in the French original), the Maxwell-Cremona correspondence (see [4] and [16]), colorings of graphs and polyhedra [20]), etc. In Section 6 we demonstrate the appropriateness of gain graphs by applying the Binary Cycle Test to generalize a theorem of Voronoi [23], that any simple tiling of $\mathbb{R}^{d}$ is the projection of a (convex) PL-surface which, given the tiling, is uniquely determined by $d+2$ free real parameters. (A spline theorist would say that the dimension of the space of $C_{1}^{0}$-splines over the tiling is $d+2$.) Such a surface is called a (convex) lifting of the tiling to $\mathbb{R}^{d+1}$. It is known that the notion of lifting and the space of liftings can be defined for any PL-realization of a homology $d$-manifold in $\mathbb{R}^{d}$. Our generalization consists in showing that the dimension of the space of liftings of any non-degenerate PL-realization in $\mathbb{R}^{d}$ of a homology $d$-manifold $\mathcal{M}$ with first homology group $\mathrm{H}_{1}\left(\mathcal{M} ; \mathbb{Z}_{2}\right)=0$ is $d+2$, provided that each $(d-3)$-cell of $\mathcal{M}$ is incident to exactly four $d$-cells. This application was chosen because in the course of the proofs gain graphs appear in a number of different geometric guises and we employ the Binary Cycle Test several times.

Another application of the Binary Cycle Test is to Voronoi's conjecture on parallelohedra, which states that any parallelohedron is an affine copy of the Dirichlet domain of a lattice. Deza and Grishukhin [6] recently gave a number of combinatorial reformulations of Voronoi's conjecture that are essentially gain-graphical. The proof of the Voronoi conjecture can be reduced to a number of questions about a certain type of finite gain graph with gain group $\mathbb{R}^{*}$, called the Venkov graph. (See [14], [17], [18], and [6] for definitions and examples.) We have noticed that the Binary Cycle Test simplifies attacking these questions, because in the case of Venkov graphs it is much easier to look 
for a basis of the binary cycle space rather than of the integral cycle space. Very recently Ordine [14] made a major advance in Voronoi's conjecture by proving an important special case, where the dual figures for the stars of $(d-3)$-cells of the tiling are restricted to tetrahedra, octahedra, and pyramids. The Binary Cycle Test can be applied to simplify his proof greatly; we hope to explain this elsewhere.

\section{Preliminaries}

Our graphs may be infinite. Loops (edges with two equal endpoints) and multiple edges are allowed. We assume that $\Gamma$ is connected and that it has a fixed (but arbitrary) orientation.

A circle $C$ can be written as the edge set of a simple closed walk in a way that is unique up to the choice of initial vertex and direction. Then it depends only on $C$, not on the choice of the walk, whether the walk is in Kerg. If it is, we say $C$ is balanced.

If $\widetilde{w}$ is a closed walk on $\Gamma$, denote by $\mathbf{w}$ the corresponding element $\widetilde{w}^{a}$ of $Z_{1}(\Gamma)$. If $\mathbf{w} \in Z_{1}(\Gamma)$, denote by $[\mathbf{w}]$ the corresponding element of $Z_{1}\left(\Gamma ; \mathbb{Z}_{2}\right)$.

We denote by $\pi(\Gamma)$ the fundamental group of $\Gamma$. The essential gain group of a gain graph is $g(\pi(\Gamma))$. It is well defined up to conjugation in $G$. Evidently, $(\Gamma, g, G)$ is balanced if and only if the essential gain group is trivial.

Naturally, $g$ induces a homomorphism from $\pi(\Gamma)$ to $G$ and from $\mathbb{Z} E$, the free abelian group on $E$, to $G / G^{\prime}$, where, as usual, $G^{\prime}$ denotes the commutator subgroup. Hence, $g$ induces a homomorphism from $Z_{1}(\Gamma)$, the abelianization of $\pi(\Gamma)$, to $G / G^{\prime}$.

A cyclic orientation, or lifting to the fundamental group, of an element $b$ of $Z_{1}\left(\Gamma ; \mathbb{Z}_{2}\right)$ is any element $\widetilde{b}$ of $\pi(\Gamma)$ whose abelianization modulo 2 is $b$. A circle has natural cyclic orientations obtained by taking an element of $\pi(\Gamma)$ corresponding to a simple walk around the circle in either direction from any starting point. A lifting of $b$ to the cycle space is the image $\mathbf{b}$ in $Z_{1}(\Gamma)$ of a cyclic orientation of $b$. If $B$ is a basis of $Z_{1}\left(\Gamma ; \mathbb{Z}_{2}\right)$, then denote by $\widetilde{B}$ a cyclic orientation of $B$. As one can see from our notation, if $b$ denotes an element of the binary cycle space, then $\mathbf{b}$ denotes one of the possible liftings of $b$ to the cycle space, and $\widetilde{b}$ is one of the liftings of $b$ to the fundamental group.

If $H \leq \pi(\Gamma)$, then $H^{a}$ denotes $H /\left(\pi(\Gamma)^{\prime} \cap H\right)$, the abelianization of $H$ with respect to $\pi(\Gamma)$; that is, $H^{a}$ is the image of $H$ in $Z_{1}(\Gamma)$. If $L, M \leq A$, we denote by $L / M$ the image of $L$ in $A / M$; in particular, $L / 2 A$ is $L$ reduced modulo 2.

\section{The Test for Abelian Groups}

Lemma 3.1. Let $A$ be a free abelian group, and let $h$ be a homomorphism from A to $G$, an abelian group without odd torsion. Let $L$ be a subgroup of $A$ such that $h(L)=0$ and $L / 2 A=A / 2 A$. Suppose $K$ is a subgroup of $A$ such that $K /(L \cap K)$ does not have elements of infinite order. Then $h(K)=0$.

Proof. Suppose there is $\mathbf{y} \in K$ such that $h(\mathbf{y}) \neq 0$. Since $h(L)=0, \mathbf{y} \notin L$. There is a minimal $l>1$ such that $l \mathbf{y} \in L$. Let $l=2^{e} a$, where $a$ is odd. Denote $a \mathbf{y}$ by $\mathbf{x}$. Notice that $e>0$, since otherwise $h(\mathbf{x})$ would have odd order in $G$. 
Now, $h(a \mathbf{y}) \neq 0$, since otherwise $h(\mathbf{y})$ would have odd order in $G$. Then $2^{e}$ is the order of $\mathbf{x}$ in $K /(L \cap K)$ and $e=\min \left\{k \mid 2^{k} \mathbf{x} \in L\right\}$. Let $\left\{\mathbf{b}_{i}\right\}$ be a basis of $L$, so that $2^{e} \mathbf{x}=\sum l_{i} \mathbf{b}_{i}$. Since $L / 2 A=A / 2 A,\left\{\left[\mathbf{b}_{i}\right]\right\}_{i}$ generates $A / 2 A$. Thus $[\mathbf{x}]=\sum \lambda_{i}\left[\mathbf{b}_{i}\right]$, i.e., $\mathbf{x}=\sum \lambda_{i} \mathbf{b}_{i}+2 \mathbf{z}$ for some $\mathbf{z} \in A$. We have $2^{e} \mathbf{x}=\sum \lambda_{i} 2^{e} \mathbf{b}_{i}+2^{e+1} \mathbf{z}$. Thus $\sum\left(l_{i}-\lambda_{i} 2^{e}\right) \mathbf{b}_{i} \in 2 A$ and all $l_{i}$ are divisible by 2 , i.e., $l_{i}=2 m_{i}, m_{i} \in \mathbb{Z}$. Since $e>0$ we have $2^{e-1} \mathbf{x}=\sum m_{i} \mathbf{b}_{i} \in L$. This contradicts the choice of $e$ as $\min \left\{k \mid 2^{k} \mathbf{x} \in L\right\}$. Therefore $h(K)=0$.

Lemma 3.2. Let $(\Gamma, g, G)$ be a gain graph, where $G$ is an abelian group without odd torsion, and let $K$ be a subgroup of $Z_{1}(\Gamma)$. If a basis $B$ of $Z_{1}\left(\Gamma ; \mathbb{Z}_{2}\right)$ has some cyclic orientation $\widetilde{B}$ that lies in the kernel of $g$, and if $K /\left(\langle\widetilde{B}\rangle^{a} \cap K\right)$ does not have elements of infinite order, then any lifting of $K$ to $\pi(\Gamma)$ lies in $\operatorname{Kerg}$.

Proof. Denote $\langle\widetilde{B}\rangle^{a}$ by $L$. The homomorphism $g$ induces, in a natural way, a homomorphism $h$ from $Z_{1}(\Gamma)$ to $G$. Obviously, $h(L)=0$ and $L / 2 Z_{1}(\Gamma)=Z_{1}(\Gamma) / 2 Z_{1}(\Gamma)=$ $Z_{1}\left(\Gamma ; \mathbb{Z}_{2}\right)$. We can now apply Lemma 3.1 and conclude that $K$ lies in the kernel of $h$. Since $G$ is abelian, any lifting of $K$ to $\pi(\Gamma)$ also lies in Kerg.

Theorem 3.3. Suppose $\Gamma$ is a finite graph and $G$ is an abelian group without odd torsion. If all elements of a basis $B$ of $Z_{1}\left(\Gamma ; \mathbb{Z}_{2}\right)$ have cyclic orientations that lie in Kerg, then $(\Gamma, g, G)$ is balanced.

Proof. Since $\Gamma$ is finite, $\operatorname{rank}\langle\widetilde{B}\rangle^{a}=\operatorname{rank} Z_{1}(\Gamma)$, so all elements of $Z_{1}(\Gamma) /\langle\widetilde{B}\rangle^{a}$ have finite order. Now apply Lemma 3.2 with $K=Z_{1}(\Gamma)$.

Lemma 3.4. Let $G$ be an abelian group without odd torsion. If some basis $B$ of $Z_{1}\left(\Gamma ; \mathbb{Z}_{2}\right)$ has a cyclic orientation $\widetilde{B}$ that lies in $\operatorname{Ker} g$, then $g(\pi(\Gamma))$ is 2-divisible.

Proof. The elements of $Z_{1}(\Gamma)$ that have finite order in $Z_{1}(\Gamma) /\langle\widetilde{B}\rangle^{a}$ form a subgroup in $Z_{1}(\Gamma)$, which we denote by $F$. Since $F /\langle\widetilde{B}\rangle^{a}$ does not have elements of infinite order, Lemma 3.2 with $K=F$ implies that $g(F)=0$. Thus if $\mathbf{y}-\mathbf{x} \in F, g(\mathbf{y}-\mathbf{x})=0$. Therefore, $g$ induces an epimorphism $h$ from $Z_{1}(\Gamma) / F$ onto $g(\pi(\Gamma))$.

Let $\mathbf{y} \in Z_{1}(\Gamma)$. Since $\langle\widetilde{B}\rangle^{a} \subset F$ and $\left[\langle\widetilde{B}\rangle^{a}\right]=Z_{1}\left(\Gamma ; \mathbb{Z}_{2}\right)$, there is $\mathbf{x} \in F$ such that $\mathbf{y}-\mathbf{x}=2 \mathbf{z}$ for some $\mathbf{z} \in Z_{1}(\Gamma)$. Thus $\mathbf{y} \in 2 \mathbf{z}+F$, and $\mathbf{y}+F=2 \mathbf{z}+F=2(\mathbf{z}+F)$ as cosets. Therefore all elements of $Z_{1}(\Gamma) / F$ are 2-divisible.

Let $a \in g(\pi(\Gamma))$. Since the homomorphism $h$ from $Z_{1}(\Gamma) / F$ is onto $g(\pi(\Gamma))$, there is $\mathbf{y} \in Z_{1}(\Gamma) / F$ such that $h(\mathbf{y})=a$. Since $\mathbf{y}$ is 2-divisible, $a$ is also 2-divisible: $a=$ $h(\mathbf{y})=h(2 \mathbf{z})=2 h(\mathbf{z})$.

Theorem 3.5. Let $G$ be an abelian group, without odd torsion, which has no infinitely 2-divisible elements other than zero. If some basis $B$ of $Z_{1}\left(\Gamma ; \mathbb{Z}_{2}\right)$ has a cyclic orientation $\widetilde{B}$ that lies in $\operatorname{Ker} g$, then $(\Gamma, g, G)$ is balanced.

Proof. If $Z_{1}(\Gamma) /\langle\widetilde{B}\rangle^{a}$ does not have elements of infinite order, then an application of Lemma 3.2 with $K=Z_{1}(\Gamma)$ implies that $(\Gamma, g, G)$ is balanced. If $Z_{1}(\Gamma) /\langle\widetilde{B}\rangle^{a}$ has 
elements of infinite order, then by Lemma $3.4 g(\pi(\Gamma))$ is 2-divisible, which is only possible if $g(\pi(\Gamma))=0$. Thus $(\Gamma, g, G)$ is balanced.

Corollary 3.6. Suppose $\Gamma$ is a finite graph and $G$ is a free group. If all elements of a basis $B$ of $Z_{1}\left(\Gamma ; \mathbb{Z}_{2}\right)$ have cyclic orientations that lie in $\operatorname{Ker} g$, then $(\Gamma, g, G)$ is balanced.

Proof. $g$ induces a homomorphism $\bar{g}$ from $\pi(\Gamma)$ into $G / G^{\prime} . G / G^{\prime}$ is a free abelian group and, therefore, does not have torsion or infinitely 2-divisible elements. By Theorem $3.5, \operatorname{Im} \bar{g}=0$. However, this can only happen if $\operatorname{Im} g=1$, because Ker $g$ is a free group whose abelianization is $\operatorname{Ker} \bar{g}$.

Corollary 3.7. Let $G$ be a group such that $G / G^{\prime}$ has no torsion or has only 2-torsion. Let $(\Gamma, g, G)$ be a gain graph whose gain group is $G$. If there is a basis $B$ of the binary cycle space $Z_{1}\left(\Gamma ; \mathbb{Z}_{2}\right)$ such that for each $b \in B$ there is a cyclic orientation $\widetilde{b}$ for which $g(\widetilde{b}) \in G^{\prime}$, then every circle has gain in $G^{\prime}$.

\section{Counterexamples}

In discrete geometry the case of an infinite gain graph with an abelian 2-divisible group of gains, such as $\mathbb{R}^{n}$ or $\mathbb{R}^{*}$, is one of the most typical ones (see [20] and [16]). In such applications the cycle basis for which we can verify the balance property normally consists of circles. Might the Circle Test, i.e., the Binary Cycle Test where all elements of the binary basis are known to be circles, work for infinite graphs with 2-divisible groups of gains? Unfortunately, not. We construct an infinite gain graph with a 2-divisible, torsion-free group of gains, unbalanced yet having a binary circle basis all of whose elements are balanced.

Example 4.1. The graph is shown in Fig. 1. We use the following notation for elements of $Z_{1}(\Gamma)$ :

$$
\mathbf{e}_{k}=D_{k} L_{k} D_{k+1} U_{k} D_{k}, \quad \mathbf{g}_{k}=L_{k} L_{k+1} D_{k+1} L_{k}, \quad \mathbf{h}_{k}=U_{k} D_{k+1} U_{k+1} U_{k} .
$$

Then $\left\{\mathbf{e}_{k}, \mathbf{g}_{k}, \mathbf{h}_{k}\right\}_{k \geq 1}$ is an integral circle basis of $\Gamma$. As usual, we denote an element of $Z_{1}\left(\Gamma ; \mathbb{Z}_{2}\right)$ corresponding to $\mathbf{x} \in Z_{1}(\Gamma)$ by $[\mathbf{x}]$. Let

$$
\mathbf{B}=\left\{\mathbf{e}_{k}+\mathbf{g}_{k}+\mathbf{h}_{k}\right\}_{k \geq 1} \cup\left\{\mathbf{g}_{k}+\mathbf{e}_{k+1}\right\}_{k \geq 1} \cup\left\{\mathbf{h}_{k}+\mathbf{e}_{k+1}\right\}_{k \geq 1}
$$

and let $B=\{[\mathbf{b}] \mid \mathbf{b} \in \mathbf{B}\}$. $B$ is obviously a basis of $Z_{1}\left(\Gamma ; \mathbb{Z}_{2}\right)$. Let $H$ denote the subgroup of $Z_{1}(\Gamma)$ (hence of $\mathbb{Z} E$ ) generated by $\mathbf{B}$, and let $\chi: \mathbb{Z} E \rightarrow \mathbb{Z} E / H$ be the quotient map. Now, we set the gain group to be $\mathbb{Z} E / H$ and the gain map $g$ to be $\chi a$, where $a$ is the abelianization mapping from $F_{E}$ to $\mathbb{Z} E$. Then the essential gain group $g(\pi(\Gamma))=\chi\left(Z_{1}(\Gamma)\right)=Z_{1}(\Gamma) / H$. By construction, $B$ is a basis of the binary cycle space that consists of balanced circles. If $H \neq Z_{1}(\Gamma)$, then the constructed gain graph is not balanced, because $Z_{1}(\Gamma) / H=\left\langle\chi\left(\mathbf{e}_{k}\right), \chi\left(\mathbf{g}_{k}\right), \chi\left(\mathbf{h}_{k}\right) \mid k \geq 1\right\rangle=g(\pi(\Gamma))$. However, in fact, $H \neq Z_{1}(\Gamma)$, because there is no finite linear combination of elements of $H$ that is equal to $\mathbf{e}_{1}$. 


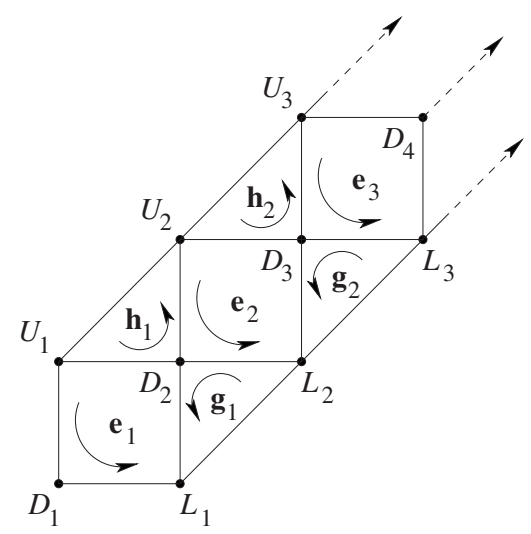

Fig. 1. Counterexample to the Circle Test with infinitely 2-divisible elements.

Denote by $\mathbb{Z}\left[\frac{1}{2}\right]$ the ring of dyadic rationals, i.e., the ring of rational numbers whose denominators are powers of 2 . Its additive group $\mathbb{Z}\left[\frac{1}{2}\right]^{+}$is the unique smallest group that contains an infinitely 2 -divisible element of infinite order. One can show that $Z_{1}(\Gamma) / H \cong$ $\mathbb{Z}\left[\frac{1}{2}\right]^{+}$by mapping $\mathbf{e}_{i}+H \mapsto d_{i}$ where $\left\{d_{i}\right\}_{i=1}^{\infty}$ generates $\mathbb{Z}\left[\frac{1}{2}\right]^{+}$and satisfies $d_{i}=2 d_{i+1}$. Thus, the essential gain group $g(\pi(\Gamma))$ is 2-divisible.

Notice that a circle $\mathbf{e}_{i}+\mathbf{g}_{i}+\mathbf{h}_{i}$ has five edges.

Conjecture 4.1. Let $G$ be a torsion-free abelian group, possibly 2-divisible, and suppose $Z_{1}(\Gamma)$ has a basis that consists of balanced circles with at most $k$ edges. Then $(\Gamma, g, G)$ is balanced if $k=3$, but it need not be balanced if $k=4$.

There are also finite counterexamples, but the gain group necessarily has an element of odd order. Two such examples, based respectively on the wheel and on an even circle with all edges doubled, are found in [19, Theorem 6.16].

\section{Algebra of Gain Graphs and Their States}

In applications of the kind in Section 6 gain graphs are normally used in the following setup. Let $(\Gamma, g, G)$ be a gain graph. Let $Q$ be a set, which we call the set of qualities, and suppose $G$ acts on $Q$; for $q \in Q$ and $h \in G$ we denote the result of the action by $[q] h$. For instance, it may be that $Q=G$ and the action is by right multiplication (the right regular action). The combination $(\Gamma, g, G, Q)$ is a permutation gain graph. (The idea was introduced by Gross and Tucker [8] under the name "permutation voltage graph".) A state of the graph is an assignment $s$ of qualities to the vertices of $\Gamma$. We want to find a state such that for any two adjacent vertices $v$ and $w$, their qualities $s(v)$ and $s(w)$ are connected by $s(w)=[s(v)] g(v w)$. Such a state is called satisfied with respect to $g$, or $g$-satisfied. The set of all $g$-satisfied states is denoted by $\operatorname{Sat}_{g}(\Gamma, G, Q)$. 
The set of all states that are satisfied with respect to at least one element of $G$ is denoted by $\operatorname{Sat}(\Gamma, G, Q)$. If $H$ is a subgroup of $G^{E}$, then $\operatorname{Sat}_{H}(\Gamma, G, Q)$ is the set of all states that are satisfied with respect to some $g \in H$. A $g$-satisfied state always exists if $g$ is balanced, because in this case one can arbitrarily choose the state of one vertex in each connected component, then assign the states of other vertices according to the rule of satisfaction. The exact rule is that if $s\left(v_{0}\right)$ is the arbitrary state and $v$ is a vertex connected to $v_{0}$ by a walk $W$, then $s(v)=\left[s\left(v_{0}\right)\right] g(W)$.

The set $G^{E}$ of all gain maps with values in $G$ forms a group under componentwise multiplication, $g g^{\prime}(e)=g(e) g^{\prime}(e)$. If $G$ is abelian the set $\mathrm{Bal}(\Gamma, G)$ of all balanced gain maps is a subgroup of $G^{E}$. If, in addition, $\Gamma$ is connected, then $|\operatorname{Bal}(\Gamma, G)|=|G|^{|V|-1}$, and $\operatorname{Bal}(\Gamma, G) \cong G^{|V|-1}$ if $G$ is abelian, because one can assign gains arbitrarily to the edges of a spanning tree and then the other edge gains are uniquely determined by balance. In general, $\operatorname{Bal}(\Gamma, G) \varsubsetneqq G^{|V|-1}$ if $G$ is non-abelian. From now on we assume that $\Gamma$ is connected.

If $Q$ is a group, the set $Q^{V}$ of states forms a group under componentwise multiplication, $s s^{\prime}(v)=s(v) s^{\prime}(v)$. From now on we assume that $Q$ is a group. We do not assume, in general, that the action of $G$ on $Q$ is a group action.

If $G$ does act on $Q$ in the manner of a group action (i.e., through a homomorphism $G \rightarrow$ Aut $Q$ ), then the set of all $g$-satisfied states, $\operatorname{Sat}_{g}(\Gamma, G, Q)$, forms a subgroup of $Q^{V}$. If in addition $\Gamma$ is connected and $g$ is balanced, then

$$
\operatorname{Sat}_{g}(\Gamma, G, Q) \cong Q
$$

because the choice of $s(v)$ at one vertex $v$ determines $s$.

Another way $G$ may act on $Q$ is by a product action, that is, where

$$
\left[q q^{\prime}\right]\left(f f^{\prime}\right)=([q] f)\left(\left[q^{\prime}\right] f^{\prime}\right) .
$$

Affine action of $\mathbb{R}^{n}$ on itself (by translations) is an example of a product action in geometry. A product action is very restricted.

Lemma 5.1. An action of $G$ on $Q$ is a product action if and only if it has the form $[q] f=\sigma(q) c(f)$, where $\sigma: Q \rightarrow Q$ is an endomorphism of $Q$ and $c: G \rightarrow Q$ is a homomorphism of $G$ into the center of $Q$.

Proof. Any action of this form is clearly a product action. For the converse, compare the two sides of (5.1). First, with $q=q^{\prime}=1$; this establishes the existence of a homomorphism $c$ given by $c(f)=[1] f$. Next, with $q^{\prime}=1$ and $f=1$; this establishes that $[q] f^{\prime}=([q] 1) \cdot c\left(f^{\prime}\right)$. Taking $q=1$ and $f^{\prime}=1$ establishes that $[q] f=c(f) q^{\prime}$; combining this with the previous conclusion we get $[q] 1 \cdot c(f)=[q] f=c(f) \cdot[q] 1$ for all $q \in Q$. So, $c(f)$ is in the center of $Q$. Taking $f=f^{\prime}=1$ shows that $\sigma: q \mapsto[q] 1$ is a homomorphism $Q \rightarrow Q$.

We call $(g, s) \in G^{E} \times Q^{V}$ a satisfied pair if $s$ is a satisfied state with respect to the gain map $g$. With a product action of $G$ on $Q$, the set of satisfied pairs forms a group SatPair $(\Gamma, G, Q)$ with respect to componentwise multiplication, $(g, s)\left(g^{\prime}, s^{\prime}\right)=$ 
$\left(g g^{\prime}, s s^{\prime}\right)$. If $H \subseteq G^{E}$, then $\operatorname{SatPair}_{H}(\Gamma, G, Q)$ stands for the set of all satisfied pairs $(s, g)$ where $g \in H$. If $G$ is abelian and $H$ happens to be a subgroup of $\operatorname{Bal}(\Gamma, G)$, $\operatorname{SatPair}_{H}(\Gamma, G, Q)$ forms a subgroup of $\operatorname{SatPair}(\Gamma, G, Q)$.

We call an action of $G$ on $Q$ fixed-point free if every element of $G$, other than the identity, acts without fixed points. If $\Gamma$ is connected and the action of $G$ is fixed-point free, then $\operatorname{Sat}(\Gamma, G, Q) \cong \operatorname{Bal}(\Gamma, G) \times Q$, because, as shown in the following lemma, a gain mapping $g \in G^{E}$ and the value $q \in Q$ at one vertex together determine a satisfied state if and only if $g \in \operatorname{Bal}(\Gamma, G)$. From the definitions we have:

Lemma 5.2. Suppose the action of $G$ on $Q$ is fixed-point free. Then a permutation gain graph $(\Gamma, g, G, Q)$ has a satisfied state if and only if $(\Gamma, g, G)$ is balanced, and then the state is determined by its value at any one vertex.

On the other hand, if the action if not fixed-point free and $\Gamma$ is not a tree, then one can always define an unbalanced gain map that has a satisfied state. The lemma implies, in particular, that if $H$ is a subgroup of $\operatorname{Bal}(\Gamma, G)$, then there is a natural bijection between $\operatorname{Sat}_{H}(\Gamma, G, Q)$ and $H \times Q$.

Suppose $G$ acts on $Q$ in the manner of a group action. If $Q$ is a module over a ring $\mathcal{R}$ and the group action is linear over $\mathcal{R}$, then for any gain map $g, \operatorname{Sat}_{g}(\Gamma, G, Q)$ is also an $\mathcal{R}$-module. Furthermore, by Lemma 5.2,

$$
\operatorname{Sat}_{g}(\Gamma, G, Q) \cong Q .
$$

If $G$ is also an $\mathcal{R}$-module, then the group of gain mappings, $G^{E}$, is an $\mathcal{R}$-module. If, moreover, $H$ is a submodule of $\operatorname{Bal}(\Gamma, G)$, then the action of $G$ on $Q$ is indeed a product action, $\operatorname{SatPair}_{H}(\Gamma, G, Q)$ is a subgroup of $\operatorname{SatPair}(\Gamma, G, Q)$, and

$$
\operatorname{SatPair}_{H}(\Gamma, G, Q) \cong Q \oplus H .
$$

In particular, $\operatorname{SatPair}(\Gamma, G, Q) \cong Q \oplus G^{|V|-1}$.

If the action of module $G$ on module $Q$ is also fixed-point free, then by Lemma 5.2,

$$
\operatorname{Sat}_{H}(\Gamma, G, Q) \cong \operatorname{SatPair}_{H}(\Gamma, G, Q) \cong Q \oplus H
$$

A special case of permutation gain graphs has been studied by Joswig [11] and Izmestiev and Joswig [10] in connection with coloring of tilings of manifolds and problems in the geometric topology of covering spaces. Let $\Gamma$ be the dual graph of a finite, pure, $d$-dimensional, strongly connected simplicial complex $\Delta$ on $n$ vertices, and let $G$, the gain group, be $S_{n}$, the symmetric group on the vertices of $\Delta$. The gain mapping $g$ takes an edge connecting adjacent $d$-simplices whose symmetric difference is a pair $\{i, j\}$ to the transposition that exchanges $i$ and $j$ (Joswig calls this a "perspectivity"). Joswig calls the image $g(\pi(\Gamma))$ of the group of closed edge-walks beginning and ending at a vertex $v \in \Gamma$ the group of "projectivities" and writes it $\Pi(\Delta, v)$. The main object of Izmestiev and Joswig's studies is the size and structure of this group for various combinatorial manifolds. 


\section{Gain Graphs in Geometry}

Given a tiling of $\mathbb{R}^{d}$ by convex polyhedra, it is natural to ask whether this tiling can be thought of as the vertical projection of a convex PL-surface or, more generally, any PL-surface. A PL-function on $\mathbb{R}^{d}$ is known as a $C_{1}^{0}$-spline in approximation theory. The problem of finding the dimension of the space of such splines on a given tiling has received significant attention from discrete geometers and spline enthusiasts (see, e.g., [24] or [2] for details). Voronoi [23] showed that any simple tiling $T$, i.e., such that each vertex is incident to exactly $d+1$ tiles, is indeed the projection of a convex surface, which he called a generatrix of $T$; furthermore, a surface that gives this tiling as a projection is uniquely determined by the choice of an affine support function and a dihedral angle.

A tiling is an example of a more general concept, namely a PL-realization $M$ in $\mathbb{R}^{d}$ of a $d$-dimensional manifold $\mathcal{M}$. In this section, as an application of the Binary Cycle Test, we ask about possible PL-realizations of $\mathcal{M}$ in $\mathbb{R}^{d+1}$ that give $M$ as a vertical projection.

If $M$ is a PL-realization in $\mathbb{R}^{d}$ of a cell-complex $\mathcal{M}$ (cell-complex defined, e.g., in the sense of Seifert and Threlfall [21]; the PL-realization is what is called a geometric cell-complex), and $L$ is another PL-realization of $\mathcal{M}$, but in $\mathbb{R}^{d+1}$, such that for each cell $C$ of $\mathcal{M}$ its realization in $M$ is the vertical projection of its realization in $L$, then $L$ is called a lifting of $M$. The notion of lifting naturally generalizes that of a $C_{1}^{0}$-spline. The graph of a $C_{1}^{0}$-spline is a lifting of the tiling over which it is defined, while a lifting of a PL-realization $M$ in $\mathbb{R}^{d}$ of a $d$-manifold $\mathcal{M}$ is the graph of a $C_{1}^{0}$-spline if and only if $M$ is an embedding. Obviously, liftings can be added, just like $C_{1}^{0}$-splines. The space of liftings of $M$ is denoted by $\operatorname{Lift}(M)$. A trivial kind of lifting is to a hyperplane in $\mathbb{R}^{d+1}$. The opposite kind is a sharp lifting: we call a PL-realization in $\mathbb{R}^{d+1}$ of a $d$-complex sharp if any two adjacent $d$-cells are realized on different affine hyperplanes.

Of course, questions about projections of piecewise-linear surfaces belong to the realm of projective geometry. To make our presentation more visual and accessible we do all our geometry in the real affine space $\mathbb{R}^{d}$, although at the end of the section we give our result a proper projective interpretation.

Although some of the constructions that we treat below were considered in [16], the Binary Cycle Test is formulated incorrectly there (in Lemma 4.1). Roughly speaking, that formulation does not have any restriction on gain groups, due to the fact that the author had in mind only abelian groups. However, since the test was applied there for abelian, torsion-free groups only, all the geometric results of Rybnikov [16] about PLrealizations of (and splines over) a manifold $\mathcal{M}$ with $\mathrm{H}_{1}\left(\mathcal{M} ; \mathbb{Z}_{2}\right)=0$ hold true if one assumes that the manifold has finitely many cells; this last requirement was also omitted in [16]. (When $\mathcal{M}$ is simply connected the finiteness restriction is not necessary, since in this case there is an alternative argument, which uses the fundamental group - see [20] for proofs and examples.)

\subsection{Technical Preparation}

We use standard notions of PL-topology. (See, e.g., [13] or [21].) A regular CW-complex $\mathcal{K}$ is a locally finite $\mathrm{CW}$-complex in which all gluing mappings are homeomorphisms. A regular $\mathrm{CW}$-complex has a barycentric subdivision $\mathcal{K}^{b}$, which is a simplicial com- 
plex. $\mathcal{K}$ also has a natural dual structure, called the dual cell-decomposition; we denote the dual $\mathrm{CW}$-complex by $\mathcal{K}^{*}$. Note that $\mathcal{K}, \mathcal{K}^{*}$, and $\mathcal{K}^{b}$ have the same support and are homeomorphic. The face lattices of $\mathcal{K}$ and $\mathcal{K}^{*}$ are dual. A cell is always assumed to be relatively open and the closure of a cell need not be homeomorphic to a ball. A $k$-cell $C^{*}$ of $\mathcal{K}^{*}$ is the union of all simplices of $\mathcal{K}^{b}$ whose closures share the barycenter of a $(d-k)$-cell $C$ of $\mathcal{K}$. The boundary of $C^{*}$ is called the link of $C$. The dimension of $\mathcal{K}$ is the maximum dimension of any cell of $\mathcal{K}$. Facets of $\mathcal{K}$ are cells of dimension $\operatorname{dim} \mathcal{K}-1$, and ridges of $\mathcal{K}$ are cells of dimension $\operatorname{dim} \mathcal{K}-2$. Two $k$-cells are adjacent if their closures share a $(k-1)$-cell. A pure $d$-dimensional CWcomplex is strongly connected if any two $d$-cells can be joined by a sequence of adjacent $d$-cells.

A homology sphere is defined inductively: a homology 0-sphere is a standard 0sphere, and a higher-dimensional homology sphere is a strongly connected regular CWcomplex such that: (1) all its homology groups are like those of a standard sphere and (2) the boundaries of all cells of the dual cell-decomposition are homology spheres. A homology ball is a cone over a homology sphere. A finite homology d-manifold (with boundary) is a strongly connected regular CW-complex with finitely many cells, in which the link of each $k$-cell, for $0 \leq k<d$, is a homology sphere (or ball if the cell belongs to the boundary) of dimension $d-k-1$. Seifert and Threlfall [21] allow the cells of a homology manifold to be homology balls; then the regular CW-complexes in our definitions should be replaced by regular homology CW-complexes. All of our results hold for this more general interpretation.

If $\mathcal{K}$ is a regular $\mathrm{CW}$-complex and $r$ is a continuous map from the barycentric subdivision $\mathcal{K}^{b}$ to $\mathbb{R}^{N}$ such that (1) each abstract simplex is mapped to an affine simplex, and (2) the simplices of $\mathcal{K}^{b}$ making up a $k$-cell of $\mathcal{K}$ are mapped to the same affine $k$-flat of $\mathbb{R}^{N}$, then $K=(\mathcal{K}, r)$ is called a $P L$-realization of $\mathcal{K}$. The realization is called non-degenerate if $r$, restricted to the closure of each cell of $\mathcal{K}$, is a homeomorphism.

\subsection{Local Considerations}

An oriented facet is an ordered pair of adjacent $d$-cells. The minimum possible number of $d$-cells that make contact at a $k$-cell of a convex tiling of $\mathbb{R}^{d}$, or of a $(d+1)$-polytope, is $d-k+1$. We call the star of a $k$-cell in a manifold simple (a.k.a.primitive in the theory of parallelohedra) if the cell is incident to only $d-k+1 d$-cells. A $d$-manifold is called $k$-simple if the star of each $k$-cell is simple. A manifold with boundary is $k$-simple if the stars of all its interior $k$-cells are simple. Notice that $k$-simplicity implies $m$-simplicity for $m>k$.

The dual graph $\Gamma(\mathcal{M})$ of a homology $d$-manifold $\mathcal{M}$ (possibly with boundary) is the graph of adjacency of $d$-cells. It is the 1-skeleton of the dual cell-decomposition $\mathcal{M}^{*}$. The vertices are the $d$-cells of $\mathcal{M}$ and the edges are the pairs of $d$-cells that share a common facet. The edges of $\Gamma(\mathcal{M})$ can be thought of as the internal facets of $\mathcal{M}$. In the dual graph of a $k$-simple manifold, the subgraphs corresponding to the stars of $k$-cells are complete subgraphs on $d-k+1$ vertices. Subgraphs of $\Gamma(\mathcal{M})$ that correspond to the stars of ridges are circles; we call them ridge circles and, in the case when the stars of ridges are simple, ridge triangles. 
A reciprocal for a PL-realization $M$ in $\mathbb{R}^{d}$ of a homology manifold $\mathcal{M}$ is a rectilinear realization $R$ in $\mathbb{R}^{d}$ of the dual graph $\Gamma(\mathcal{M})$ such that the edges of $R$ are perpendicular to the corresponding facets. If none of the edges of $R$ collapse into a point, the reciprocal is called non-degenerate; a one-point reciprocal is called trivial. A reciprocal for the star of a cell $C$ is called a local reciprocal of $C$. Reciprocals were originally considered by Maxwell [12] and Cremona [5] in connection with stresses in plane frameworks. Crapo and Whiteley [4] gave a modern treatment of the Maxwell-Cremona theory of reciprocals, stresses, and liftings for 2-manifolds. Obviously, reciprocals can be added and multiplied by real scalars. Geometrically, multiplication corresponds to scaling. The addition is induced by vector addition of the vertices. Thus, for a given $M$ the reciprocals form a linear space. This space, factored by all translations of $\mathbb{R}^{d}$, is denoted $\operatorname{Rec}(M)$.

A PL-realization of a homology $d$-manifold is called generic if no pair of adjacent facets lies in the same $(d-1)$-plane.

Lemma 6.1. For each non-degenerate generic PL-realization in $\mathbb{R}^{d}$ of a simple star of a cell there is a non-degenerate reciprocal. It is unique up to translations, homotheties, and central inversions.

Proof. The existence of a non-degenerate reciprocal for a simple star is a trivial exercise in linear algebra. Such a reciprocal is the 1-skeleton of a simplex whose edges are perpendicular to the corresponding facets. Since directions of edges and the angles between them are uniquely determined by the geometry of the star, the reciprocal is unique up to affine transformations preserving directions and angles.

Lemma 6.2. If Star is a non-degenerate generic PL-realization of a simple star of a cell, then $\operatorname{dim} \operatorname{Lift}($ Star $)=d+2$.

Proof. Any three $d$-cells of Star form a triangle in the dual graph. Choose two $d$-cells in Star. By fixing two affine functions that lift the union of these $d$-cells to $\mathbb{R}^{d+1}$, so that these functions coincide on the common facet of the cells, we fix the lifting for all of Star. Since a lifting of two adjacent $d$-cells is defined by $d+2$ parameters (e.g., the coefficients of an affine function in $d$ variables and a dihedral angle in $(d+1)$-space), $\operatorname{dim} \operatorname{Lift}($ Star $)=d+2$.

\subsection{From Local to Global}

In the remainder of the section we show that, subject to the topological restriction that $\mathrm{H}_{1}\left(\mathcal{M} ; \mathbb{Z}_{2}\right)=0$ and the combinatorial restriction of $(d-3)$-simplicity, liftings of $(d-2)$-simple manifolds behave in the same way as those of simple stars. (Although a tiling of $\mathbb{R}^{d}$ may not be a CW-complex, all our theorems hold for the case when $M$ is a finite tiling.)

The following lemma is a homological version of what some call Poincaré's $(d-$ 2)-face principle [15] in discrete geometry, which, basically, says that for a group of isometries to tile a Euclidean space, sphere, or hyperbolic space with copies of a $d$ - 
polyhedron $T$ it is enough that for each $(d-2)$-face $F$ of $T$ there are group elements $g_{1}, \ldots, g_{k}$ such that $T, g_{1}(T), \ldots, g_{k}(T)$ fill the space, face-to-face, around $F$ without gaps or overlaps. See [7] for an exact formulation and recent generalization of this principle.

Lemma 6.3. Let $\mathcal{M}$ be a finite homology $d$-manifold with $d \geq 2$ and with $\mathrm{H}_{1}(\mathcal{M}$; $\left.\mathbb{Z}_{2}\right)=0$. Let $(\Gamma(\mathcal{M}), g, G)$ be an abelian gain graph whose underlying graph is $\Gamma(\mathcal{M})$ and whose gain group has no odd torsion. Then the gain graph is balanced if and only if the ridge circles of $\Gamma(\mathcal{M})$ are balanced.

Proof. The "only if" part follows from the definition of a balanced graph.

Consider the dual cell-decomposition $\mathcal{M}^{*}$ of $\mathcal{M}$. The 2-cells of this decomposition are in one-to-one correspondence with the ridges of $\mathcal{M}$. Since $\mathrm{H}_{1}\left(\mathcal{M} ; \mathbb{Z}_{2}\right)=0$ and the underlying spaces of $\mathcal{M}$ and $\mathcal{M}^{*}$ are the same, the boundaries of the 2-cells of $\mathcal{M}^{*}$ generate $Z_{1}\left(\mathcal{M}^{*} ; \mathbb{Z}_{2}\right)$. These boundaries are circles and they are balanced by our assumption. According to the Binary Cycle Test for finite graphs, $(\Gamma(\mathcal{M}), g, G)$ must be balanced.

For instance, Lemma 6.3 applies to homology spheres of dimension at least 2.

Theorem 6.4. Let $\mathcal{M}$ be a finite (d-3)-simple homologyd-manifold without boundary, withd $\geq 3$, and with $\mathrm{H}_{1}\left(\mathcal{M} ; \mathbb{Z}_{2}\right)=0$. Let $M$ be a non-degenerate generic PL-realization of $\mathcal{M}$ in $\mathbb{R}^{d}$. Then $M$ has a non-degenerate reciprocal and $\operatorname{dim} \operatorname{Rec}(M)=1$.

Proof. The main ingredients of the proof are the dual cell-decomposition $\mathcal{M}^{*}$ of $\mathcal{M}$, the notion of gain graph, and the Binary Cycle Test for balance.

Let $\Gamma=\Gamma(V, E)$ be the dual graph of $\mathcal{M}$. An oriented facet can be thought of as an oriented edge of $\Gamma$.

From the algebraic standpoint a reciprocal for $M$ is a linear operator $r$ from the vector space $\mathrm{C}^{0}\left(\Gamma, \mathbb{R}^{d}\right)=\left(\mathbb{R}^{d}\right)^{V}$ of 0 -cochains on $\Gamma(V, E)$ with values in $\mathbb{R}^{d}$, which has the property that $\delta r\left(C_{i}, C_{j}\right)$ is orthogonal to the common facet $F_{i j}$ of $C_{i}$ and $C_{j}$, where $\delta$ : $\mathrm{C}^{0}\left(\Gamma, \mathbb{R}^{d}\right) \rightarrow \mathrm{C}^{1}\left(\Gamma, \mathbb{R}^{d}\right)$ is the coboundary map, defined by $\delta r\left(C_{i}, C_{j}\right)=r\left(C_{j}\right)-r\left(C_{i}\right)$ for each oriented facet $\left(C_{i}, C_{j}\right)$. (Thus, the edges $\left\{\left(r\left(C_{i}\right), r\left(C_{j}\right)\right)\right\}$ of a non-degenerate reciprocal have a natural interpretation as the linear parts of the equations defining the facets of $M$. These linear parts are often called the covectors of the facets of $M$.) It follows from the definition that

$$
\delta r\left(C_{1}, C_{2}\right)=-\delta r\left(C_{2}, C_{1}\right)
$$

and that

$$
\delta r\left(C_{1}, C_{2}\right)+\delta r\left(C_{2}, C_{3}\right)+\delta r\left(C_{3}, C_{1}\right)=0
$$

whenever $C_{1}, C_{2}, C_{3}$ share a common ridge. Equation (6.1) means that $\delta r$ is a gain map on $\Gamma$ with gain group $\mathbb{R}^{d}$. The gain graph is balanced because $\delta r$ is a coboundary. Because $\Gamma$ is connected, $r$ is completely determined by $\delta r$ and one point $r(C)$; indeed, $r$ 
is any satisfied state of $\left(\Gamma, \delta r, \mathbb{R}^{d}, \mathbb{R}^{d}\right)$, where $\mathbb{R}^{d}$ acts on $\mathbb{R}^{d}$ by translation (that is, the right regular action). Thus,

$$
\operatorname{Rec}(M)=\{\delta r: r \text { is a reciprocal for } M\},
$$

the space of coboundaries of reciprocals.

We associate with each oriented facet $\left(C_{1}, C_{2}\right)$ a unit vector $\mathbf{n}\left(C_{1}, C_{2}\right)$, normal to the common facet of $C_{1}$ and $C_{2}$, so that $\mathbf{n}\left(C_{1}, C_{2}\right)=-\mathbf{n}\left(C_{2}, C_{1}\right)$. Henceforth, these normals are fixed.

Consider the facet graph $\Phi$, whose vertices are the (unoriented) facets of $\mathcal{M}$ and whose edges are the pairs of facets sharing ridges. Orient the edges of $\Phi$ in an arbitrary way. Take a ridge $R$ where $d$-cells $C_{1}, C_{2}, C_{3}$ make contact. Denote by $F_{i j}$ the common facet of $C_{i}$ and $C_{j}$. Up to scaling there is only one non-trivial linear combination of $\mathbf{n}\left(C_{1}, C_{2}\right), \mathbf{n}\left(C_{2}, C_{3}\right), \mathbf{n}\left(C_{3}, C_{1}\right)$ equal to zero. Let

$$
\alpha_{12} \mathbf{n}\left(C_{1}, C_{2}\right)+\alpha_{23} \mathbf{n}\left(C_{2}, C_{3}\right)+\alpha_{31} \mathbf{n}\left(C_{3}, C_{1}\right)=0
$$

be one such combination with non-zero coefficients; it exists because $M$ is a nondegenerate PL-realization. For the oriented edge $F_{12} F_{23}$ in the facet graph, define

$$
g\left(F_{12} F_{23}\right)=\alpha_{23} / \alpha_{12} .
$$

In this way we define the map $g$ on all oriented edges of the facet graph. We call $\left(\Phi, g, \mathbb{R}^{*}\right)$ the facet gain graph.

Lemma 6.5. $\left(\Phi, g, \mathbb{R}^{*}\right)$ is a balanced gain graph.

Proof. Obviously, $g\left(F_{12} F_{23}\right)$ depends only on the geometric realization $M$ of $\mathcal{M}$ and on the (fixed) choice of normals $\mathbf{n}(A, B)$ for oriented facets. It is clear that $g\left(F_{12} F_{23}\right)=$ $g\left(F_{23} F_{12}\right)^{-1}$ and

$$
g\left(F_{12} F_{23}\right) \cdot g\left(F_{23} F_{31}\right) \cdot g\left(F_{31} F_{12}\right)=1
$$

that is, $g$ is a gain map on $\Phi$ and each triangle corresponding to a ridge (a ridge triangle of $\Phi)$ is balanced. Furthermore,

$$
\mathbf{n}\left(C_{1}, C_{2}\right)+g\left(F_{12} F_{23}\right) \mathbf{n}\left(C_{2}, C_{3}\right)+g\left(F_{23} F_{31}\right) g\left(F_{12} F_{23}\right) \mathbf{n}\left(C_{3}, C_{1}\right)=0 .
$$

The dual cell-decomposition $\mathcal{M}^{*}$ of $\mathcal{M}$ can be refined in a useful way. Each 2-cell of $\mathcal{M}^{*}$ is a triangle. Let $\mathcal{M}^{\dagger}$ be the cell-complex obtained from $\mathcal{M}^{*}$ by subdividing each edge in the middle and then joining the new vertices by three new edges within each 2-cell, thereby partitioning the 2-cell into four triangles.

Since $\mathcal{M}^{*}$ and $\mathcal{M}^{\dagger}$ are identical to $\mathcal{M}$ as topological spaces,

$$
\mathrm{H}_{1}\left(\mathcal{M}^{\dagger} ; \mathbb{Z}_{2}\right)=\mathrm{H}_{1}\left(\mathcal{M}^{*} ; \mathbb{Z}_{2}\right)=\mathrm{H}_{1}\left(\mathcal{M} ; \mathbb{Z}_{2}\right)=0
$$

Moreover, $\Phi$ is a subgraph of $\operatorname{Sk}_{1}\left(\mathcal{M}^{\dagger}\right)$. Consequently, for any $c \in Z_{1}\left(\Phi ; \mathbb{Z}_{2}\right)$ there is a 2-chain $\Delta \in \mathrm{C}_{2}\left(\mathcal{M}^{\dagger} ; \mathbb{Z}_{2}\right)$ such that $c=\partial \Delta$. The chain $\Delta$ can be decomposed as 
$\Delta_{1}+\Delta_{2}$, the sum of $\Delta_{1}=\sum \Delta_{1 j}$, where each $\Delta_{1 j}$ is a triangle whose vertices are new vertices corresponding to facets of $\mathcal{M}$ making contact at a ridge, and $\Delta_{2}=\sum \Delta_{2 i}$, where each $\Delta_{2 i}$ has one vertex in $\operatorname{Sk}_{0}\left(\mathcal{M}^{*}\right)$, corresponding to a cell of $\mathcal{M}$, and two vertices that are new vertices corresponding to facets of that cell. For each $d$-cell $C$ of $\mathcal{M}$, let $\Delta_{2 C}$ be the sum of all the $\Delta_{2 i}$ having as one vertex that vertex of $\mathcal{M}^{*}$ that corresponds to $C$. Then $c=\sum \partial \Delta_{1 j}+\sum \partial \Delta_{2 C}$ where each term in the first sum is a ridge triangle of $\Phi$ and each term in the second sum is a cycle on the boundary of a $d$-cell of $\mathcal{M}$.

This shows that ridge triangles and cycles lying on the boundaries of $d$-cells generate $Z_{1}\left(\Phi ; \mathbb{Z}_{2}\right)$. We prove next that $\left(\Phi, g, \mathbb{R}^{*}\right)$ is balanced. Since $\mathcal{M}$ is $(d-3)$-simple, the boundary of each $d$-cell $C$ is $((d-1)-2)$-simple. $\Phi_{C}$, the subgraph of $\Phi$ on the vertices that belong to $C$, is the dual graph $\Gamma(\partial C)$ of the boundary cell-complex of $C$. The corresponding gain subgraph is $\left(\Phi_{C}, g, \mathbb{R}^{*}\right)$. By (6.4) the ridge circles of $\Phi_{C}$ are balanced. Since $\operatorname{dim} \partial C \geq 2$, Lemma 6.3 applied to $\partial C$ implies that $\left(\Phi_{C}, g, \mathbb{R}^{*}\right)$ is balanced and, therefore, all cyclic orientations of binary cycles of $\Phi_{C}$ have gain 1 . We have seen that binary cycles of these two types generate $Z_{1}\left(\Phi ; \mathbb{Z}_{2}\right)$. By the Binary Cycle Test for finite graphs, $\left(\Phi, g, \mathbb{R}^{*}\right)$ is balanced.

Suppose the set of qualities $Q$ is $\mathbb{R}$ and $\mathbb{R}^{*}$ acts on $\mathbb{R}$ by multiplication. The action of $\mathbb{R}^{*}$ on $\mathbb{R} \backslash 0$ is fixed-point free and therefore, by Lemma 5.2 and the obvious properties of the all-zero state, $\operatorname{Sat}_{g}\left(\Phi, \mathbb{R}^{*}, \mathbb{R}\right) \cong \mathbb{R}$. We now explain why $\operatorname{Sat}_{g}\left(\Gamma, \mathbb{R}^{*}, \mathbb{R}\right)$ can be regarded as a one-dimensional subspace of $\left(\mathbb{R}^{d}\right)^{E}$, where $E$ is the set of edges of $\Gamma$.

Fix a non-zero satisfied state $s$ of $\left(\Phi, g, \mathbb{R}^{*}, \mathbb{R}\right)$. We denote by $s\left(C_{i}, C_{j}\right)$ the value of $s$ on a vertex of $\Phi$ corresponding to a facet between $d$-cells $C_{i}$ and $C_{j}$. We construct yet another gain graph, $\left(\Gamma, h_{s}, \mathbb{R}^{d}\right)$, this time with the gain group $\mathbb{R}^{d}$. Assign to each oriented edge $C_{1} C_{2}$ of $\Gamma$ the vector $h_{s}\left(C_{1} C_{2}\right)=s\left(C_{1}, C_{2}\right) \mathbf{n}\left(C_{1}, C_{2}\right)$.

Lemma 6.6. $\left(\Gamma, h_{s}, \mathbb{R}^{d}\right)$ is a balanced gain graph.

Proof. Since $\mathbf{n}\left(C_{1}, C_{2}\right)=-\mathbf{n}\left(C_{1}, C_{2}\right)$ and $s\left(C_{1}, C_{2}\right)=s\left(C_{2}, C_{1}\right), h_{s}\left(C_{2} C_{1}\right)=$ $-h\left(C_{1} C_{2}\right)$. Since $\mathrm{H}_{1}\left(\mathcal{M}^{*} ; \mathbb{Z}_{2}\right)=\mathrm{H}_{1}\left(\mathcal{M} ; \mathbb{Z}_{2}\right)=0$, by Lemma 6.3 balance of the ridge triangles of $\Gamma$ implies balance of the gain graph. To conclude that these triangles are balanced we have to show that

$$
s\left(C_{1}, C_{2}\right) \mathbf{n}\left(C_{1}, C_{2}\right)+s\left(C_{2}, C_{3}\right) \mathbf{n}\left(C_{2}, C_{3}\right)+s\left(C_{3}, C_{1}\right) \mathbf{n}\left(C_{3}, C_{1}\right)=0 .
$$

We divide this equation by $s\left(C_{1}, C_{2}\right)$ and recall that (with indices taken modulo 3 ) $s\left(C_{i+1}, C_{i+2}\right) / s\left(C_{i}, C_{i+1}\right)=g\left(F_{i, i+1} F_{i+1, i+2}\right)$ for the star of each ridge. So, we need

$$
\mathbf{n}\left(C_{1}, C_{2}\right)+g\left(F_{12} F_{23}\right) \mathbf{n}\left(C_{2}, C_{3}\right)+g\left(F_{12} F_{31}\right) \mathbf{n}\left(C_{3}, C_{1}\right)=0 .
$$

This is equivalent to (6.5). Thus each ridge triangle is balanced, and $\left(\Gamma, h_{s}, \mathbb{R}^{d}\right)$ is balanced by the Binary Cycle Test.

We want to prove that the elements of $\operatorname{Sat}_{g}\left(\Phi, \mathbb{R}^{*}, \mathbb{R}\right)$ correspond to the reciprocals (modulo $\mathbb{R}^{d}$ ) in one-to-one fashion; hence $\operatorname{dim} \operatorname{Rec} M=1$. Our strategy is to show 
that $\operatorname{Sat}_{g}\left(\Phi, \mathbb{R}^{*}, \mathbb{R}\right) \cong \operatorname{Rec}(M)$ as real vector spaces by producing a specific isomorphism $\rho$. We define $\rho(s)=h_{s}$. It is obvious that $\rho$ is an injection and a vector-space homomorphism. Let $H$ be the image of $\rho$. Then $H \subseteq \operatorname{Bal}\left(\Gamma, \mathbb{R}^{d}\right)$ and $\operatorname{dim} H=1$.

Consider now the permutation gain graph $\left(\Gamma, h_{s}, \mathbb{R}^{d}, \mathbb{R}^{d}\right)$, where the first three components are as above and $\mathbb{R}^{d}$ acts on $\mathbb{R}^{d}$ by translation. This action is obviously fixedpoint free. Since a reciprocal is completely defined by the coordinates of its vertices, all reciprocals form a linear subspace of $\left(\mathbb{R}^{d}\right)^{V}$. A satisfied state for $\left(\Gamma, h_{s}, \mathbb{R}^{d}, \mathbb{R}^{d}\right)$ is a reciprocal $r$ whose edge lengths have been decided by the gain graph $\left(\Gamma, h_{s}, \mathbb{R}^{d}\right)$. That is, since $\left(\Gamma, h_{s}, \mathbb{R}^{d}\right)$ is balanced and connected, we can start from any point in $\mathbb{R}^{d}$ as a vertex of the reciprocal and construct all other vertices. Then $h=\delta r$. By Lemma 5.2 this construction gives all of $\operatorname{Sat}_{h_{s}}\left(\Gamma, \mathbb{R}^{d}, \mathbb{R}^{d}\right)$; varying $s$, it gives all of $\operatorname{Sat}_{H}\left(\Gamma, \mathbb{R}^{d}, \mathbb{R}^{d}\right)$. Furthermore, it shows that $H \subseteq\{\delta r \mid r$ is a reciprocal $\}$, the reciprocal coboundary space, which is $\operatorname{Rec} M$ by (6.3). The non-zero elements of $H$ correspond (bijectively) to the non-degenerate reciprocals.

Conversely, the edges of a reciprocal $r$ determine a balanced gain map $\delta r$ with values in the gain group $\mathbb{R}^{d}$. By the orthogonality property of a reciprocal and (6.2), $\delta r$ has the form $\rho(s)$ for some non-zero $s \in\left(\Phi, g, \mathbb{R}^{*}, \mathbb{R}\right)$. Therefore, $H=\{\delta r \mid$ $r$ is a reciprocal $\}=\operatorname{Rec}(M)$.

We established earlier that $\operatorname{dim}_{\mathbb{R}} H=1$. It follows that $\operatorname{dim}_{\mathbb{R}} \operatorname{Rec}(M)=1$.

Theorem 6.7. Let $\mathcal{M}$ and $M$ be as in Theorem 6.4. Then $M$ has a sharp lifting and $\operatorname{dim}_{\mathbb{R}} \operatorname{Lift}(M)$ is $d+2$.

Proof. Since $\mathcal{M}$ is $(d-2)$-simple, in any lifting of $M$ the position of a $d$-cell $C$ from the star $\operatorname{Star}(R)$ of a ridge $R$ is completely defined by the positions of the other two $d$-cells of $\operatorname{Star}(R)$. Therefore, if the lifting of $\operatorname{Star}(R)$ can be extended to a lifting of $M$, the resulting lifting of $M$ is completely defined by the lifting of $\operatorname{Star}(R)$. By Lemma 6.2, $\operatorname{dim} \operatorname{Lift}(M) \leq d+2$.

We now prove that $\operatorname{dim} \operatorname{Lift}(M)=d+2$. Let $H$ be as in the above theorem, i.e., let $H$ be the subspace of $\operatorname{Bal}\left(\Gamma, \mathbb{R}^{d}, \mathbb{R}^{d}\right)$ that consists of those realizations of the dual graph $\Gamma$ of $\mathcal{M}$ that are reciprocals of $M$. Consider gain graphs where the underlying graph is the dual graph $\Gamma$ and the gain group is the additive group $A_{d}$ of all affine functions on $\mathbb{R}^{d}$ (this is isomorphic to $\mathbb{R}^{d+1}$ ). $A_{d}$ acts on itself by addition and this action is fixed-point free. Therefore, by Lemma 5.2, the liftings of $M$ can be identified with all satisfied states of permutation gain graphs $\left(\Gamma, h_{1}, \mathbb{R}^{d+1}, A_{d}\right)$ where $h_{1}$ is taken from the subgroup $H_{1}$ of $\operatorname{Bal}\left(\Gamma, \mathbb{R}^{d+1}\right)$ that consists of gain maps of the form $h_{1}(F)=\mathbf{e}_{F} \cdot\left(\mathbf{x}-\mathbf{c}_{F}\right)$, where $F$ is an oriented facet, $\mathbf{e}_{F}$ is the corresponding oriented edge in some reciprocal, $\mathbf{c}_{F}$ is any point on $F$, and $\mathbf{x}$ is the variable vector. This gain map can be written as $h_{1}(F)=h(F)+c(F)$, where $h(F)=\mathbf{e}_{F} \cdot \mathbf{x}$ is a gain map in $H \subseteq \operatorname{Bal}\left(\Gamma, \mathbb{R}^{d}\right)$. We know that $H \cong \mathbb{R}$. Therefore, a state $s$ of $\left(\Gamma, h_{1}, \mathbb{R}^{d+1}, A_{d}\right)$ corresponds to a lifting if and only if the gain map $c(F)=-\mathbf{e}_{F} \cdot \mathbf{x}$ is balanced. It is easy to see that all cycles of $\Gamma$ corresponding to ridges of $\mathcal{M}$ are balanced with respect to this gain map. These cycles generate all of $\mathrm{H}_{1}\left(\mathcal{M}^{*} ; \mathbb{Z}_{2}\right)=\mathrm{H}_{1}\left(\mathcal{M} ; \mathbb{Z}_{2}\right)$. Therefore, by the Binary Cycle Test $c$ is balanced. Thus, by (5.4), $\operatorname{Lift}(M) \cong A_{d} \oplus \mathbb{R} \cong \mathbb{R}^{d+2}$. 
Corollary 6.8. Let $\mathcal{M}$ be a finite simple homology d-manifold, where $d \geq 3$, such that $\mathrm{H}_{1}\left(\mathcal{M}, \mathbb{Z}_{2}\right)=0$. Let $M$ be a non-degenerate generic PL-realization in $\mathbb{R}^{d}$ whose d-cells are convex. Then $M$ is a projection of a convex polyhedron in $\mathbb{R}^{d+1}$.

Proof. Since $\mathcal{M}$ is $(d-3)$-simple, there is a sharp lifting $L$ of $M$ to $\mathbb{R}^{d+1}$. Since all cells of $\mathcal{M}$ are homeomorphic to convex polytopes, and there are $d-k+1 d$-cells making contact at each $k$-face, $\mathcal{M}$ is a topological manifold. The sharp lifting $L$ is then a simple PL-surface in $\mathbb{R}^{d+1}$, realizing the abstract manifold $\mathcal{M}$. $L$ must be an immersion, since each cell is embedded and the star of each vertex is embedded. So, we have a realization of a $d$-manifold in $\mathbb{R}^{d+1}$ which has the following properties: (1) it is an immersion, (2) it is locally (non-strictly) convex at each point, and (3) it is strictly convex at each vertex. By Van Heijenoort's [22] theorem $L$ is the boundary of a convex body. Since $\mathcal{M}$ is finite and $L$ is piecewise-linear, $L$ is a convex polyhedron.

Although Theorem 6.7 is formulated for vertical projections, it, indeed, holds for a central projection from $\mathbb{R}^{d+1}$ to $\mathbb{R}^{d} \subset \mathbb{R}^{d+1}$. It also holds for a central projection from the origin of $d$-manifolds realized in $\mathbb{R}^{d+1}$ to a $d$-sphere in $\mathbb{R}^{d+1}$ (see [16]), or projection from $(0, \ldots, 0,-1)$ of $d$-manifolds realized in $\mathbb{R}^{d+1}$ to the Minkowski hyperboloid in $\mathbb{R}^{d+1}$. Of course, the reason for such universality is that the theorem is a theorem of projective geometry. The projective formulation is Theorem 6.9. There, orthogonality between the edges of the reciprocal and facets of $M$ is replaced by duality between lines and subspaces of codimension 1 in $\mathbb{R} P^{d}$. Other terminology is essentially as in the affine version.

Theorem 6.9. Let $\mathcal{M}$ be a finite $(d-3)$-simple homology d-manifold with $\mathrm{H}_{1}(\mathcal{M}$, $\left.\mathbb{Z}_{2}\right)=0$. Let $M$ be a non-degenerate generic piecewise-linear realization in $\mathbb{R} P^{d}$. Then $M$ has a sharp piecewise-linear realization $L$ in $\mathbb{R} P^{d+1}$ that is projectively equivalent to $M$. Such a realization is uniquely determined by choosing $d+2$ free real parameters.

\section{Acknowledgment}

We thank Keith Dennis and Kenneth S. Brown for very helpful discussions.

\section{References}

1. A. D. Alexandrov (1954). On filling a space by polyhedra. (In Russian.) Vestnik Leningrad Univ. Ser. Mat. Fiz. Him. 9, 33-43. Reprinted in Selected Works. Part I: Selected Scientific Papers. Classics of Soviet Mathematics, 4. Gordon and Breach, Amsterdam, 1996.

2. L. Billera (1989). The algebra of continuous piecewise polynomials. Adv. Math. 76, 170-183.

3. H. S. M. Coxeter (1934). Discrete groups generated by reflections. Ann. of Math. 35, 588-621. Reprinted in Kaleidoscopes: Selected Writings of H. S. M. Coxeter, F. A. Sherk et al., eds. Wiley, New York, 1995.

4. H. Crapo and W. Whiteley (1993). Autocontraintes planes et polyèdres projetés. I. Le motif de base. [Plane self stresses and projected polyhedra. I. The basic pattern.] Structural Topology 20, 55-78. 
5. L. Cremona (1890). Graphical Statics. Oxford University Press, London. English translation of 1872 Italian original.

6. M. Deza and V. Grishukhin (submitted). Properties of parallelotopes equivalent to Voronoi's conjecture. On the Web at arxiv. org math.MG/0307170.

7. N. P. Dolbilin (2000). The extension theorem. Discrete Math. 221, 43-59.

8. J. L. Gross and T. W. Tucker (1977). Generating all graph coverings by permutation voltage assignments. Discrete Math. 18, 273-283.

9. J. L. Gross and T. W. Tucker (1987). Topological Graph Theory. Wiley, New York. Reprinted with minor additions: Dover, Mineola, NY, 2001.

10. I. Izmestiev and M. Joswig (2003). Branched coverings, triangulations, and 3-manifolds. Adv. Geom. 3(2), $191-225$

11. M. Joswig (2002). Projectivities in simplicial complexes and colorings of simple polytopes. Math. Z. 240(2), 243-259.

12. J. C. Maxwell (1864). On reciprocal figures and diagrams of forces. Philos. Mag. (4) 27, 250-261.

13. J. R. Munkres (1984). Elements of Algebraic Topology. Addison-Wesley, Menlo Park, CA.

14. A. Ordine (2002). Tilings by reducible and irreducible parallelotopes. In Janos Bolyai Conference on Hyperbolic Geometry (Budapest, 8 July 2002), Abstracts of Talks, pp. 43-44. To appear in Ph.D. thesis, Queen's University, Ontario.

15. H. Poincaré (1882). Théorie des groupes fuchsiens. Acta Math. 1, 1-62.

16. K. Rybnikov (1999). Stresses and liftings of cell-complexes. Discrete Comput. Geom. 21, 481-517.

17. K. A. Rybnikov (2002). Gain graphs and tilings of manifolds. In János Bolyai Conference on Hyperbolic Geometry (Budapest, 8 July 2002), Abstracts of Talks, p. 44.

18. K. A. Rybnikov (2002). On the state of Voronoi's conjecture on parallelohedra. Abstract 979-52-142 (from meeting of the Amer. Math. Soc., Boston, October 2002). Abstracts Amer. Math. Soc. 23(4), 498

19. K. A. Rybnikov and T. Zaslavsky (to appear). Cycle and circle tests of balance in gain graphs: forbidden minors and their groups. J. Graph Theory. On the Web at arXiv. org math.CO/0209316.

20. S. S. Ryshkov and K. A. Rybnikov, Jr. (1997). The theory of quality translations with applications to tilings. European J. Combin. 18, 431-444.

21. H. Seifert and W. Threlfall (1980). Seifert and Threlfall: A Textbook of Topology. Academic Press, New York. (English translation of 1934 German original.)

22. J. Van Heijenoort (1952). On locally convex manifolds. Comm. Pure Appl. Math. 5, 223-242.

23. G. F. Voronoi (1908). Nouvelles applications des paramètres continus à la théorie des formes quadratiques. Deuxième mémoire: Recherches sur les parallélloèdres primitifs. J. Reine Angew. Math. 134, 198-287.

24. W. Whiteley (1996). Some matroids from discrete applied geometry. In Matroid Theory (Seattle, Wash., 1995), J. Bonin et al., eds., pp. 171-312. Contemporary Mathematics, Vol. 197. American Mathematical Society, Providence, RI.

25. T. Zaslavsky (1989). Biased graphs. I. Bias, balance, and gains. J. Combin. Theory Ser. B 47, 32-52.

26. T. Zaslavsky (1998). A mathematical bibliography of signed and gain graphs and allied areas. Electron. J. Combin. 5, Dynamic Surveys in Combinatorics, No. DS8 (electronic).

Received December 20, 2003, and in revised form January 18, 2005. Online publication May 20, 2005. 